\title{
¿PERCIBEN DEL MISMO MODO ALUMNADO Y PROFESORADO EL DESARROLLO DE COMPETENCIAS DOCENTES? EL CASO DE UN GRADO DE CIENCIAS DE LA ACTIVIDAD FÍSICA Y EL DEPORTE
}

Do students and tutors perceive the same about developing teacher competencies? The case of a Physical Activity and Sports Science Degree

Percebem da mesma forma alunos e professores o desenvolvimento das competências docentes? O caso de um curso de ciências da atividade física e do esporte

Este estudio se ha llevado a cabo dentro del proyecto de I+D+i: “La competencias docentes en la formación inicial del profesorado de educación física”. Convocatoria de noviembre de 2013 del Programa Estatal de Investigación, Desarrollo e Innovación Orientada a los Retos de la Sociedad, en el marco del Plan Estatal de Investigación Científica y Técnica y de Innovación 2013-2016. Referencia: EDU 2013-42024-R. Duración: 3 años (2014-2016).

\section{Eloísa Lorente-Catalán (1)}

Ma José Montilla Reina (2)

\section{Alfredo Joven Pérez (3)}

\section{Albert Graells Noguerola (4)}

\section{David Castel Vilalta (5)}

(1) Institut Nacional d’Educació Física de Catalunya (INEFC), Universitat de Lleida (UdL), España. Teléfono: +34 972 272022. Correo electrónico: elorente@inefc.es

(2) Institut Nacional d’Educació Física de Catalunya (INEFC), Universitat de Lleida (UdL), España. Correo electrónico: mjmontilla@inefc.es

(3) Institut Nacional d’Educació Física de Catalunya (INEFC), Universitat de Lleida (UdL), España. Correo electrónico: ajoven@inefc.es

(4) Institut Nacional d’Educació Física de Catalunya (INEFC), Universitat de Lleida (UdL), España. Correo electrónico: albert.gn93@hotmail.com

(5) Institut Nacional d’Educació Física de Catalunya (INEFC), Universitat de Lleida (UdL), España. Correo electrónico: davidcastelvilalta@hotmail.com

¿Perciben del mismo modo alumnado y profesorado el desarrollo de competencias docentes? El caso de un grado de ciencias de la actividad física y el deporte 


\title{
Resumen
}

La competencia docente hace referencia a la capacidad del profesorado para resolver con éxito las exigencias complejas que se le planteen en el contexto educativo. Estas competencias deben ser adquiridas, en parte, durante la formación inicial del profesorado. Esta investigación pretende comparar como perciben el profesorado y alumnado el desarrollo de competencias docentes en un grado de Ciencias de la Actividad Física y el Deporte. Para ello, se pasó en el curso 2014-15 una escala de valoración autoadministrada a 65 estudiantes de $4^{\circ}$ curso y otra a 29 profesores/as. Existen diferencias significativas en la percepción del profesorado y alumnado en 24 de las 46 competencias del cuestionario. En todas las competencias el alumnado proporcionó una puntuación media más elevada que el profesorado, excepto en 9 competencias transversales. Resulta necesario seguir profundizando en las razones de esta diferencia de percepción realizando entrevistas y grupos focales que aporten información más cualitativa.

Palabras clave: Competencias docentes; formación inicial del profesorado; educación física

\begin{abstract}
Teacher competence refers to the teacher's capacity to successfully solve the complex demands placed on the educational context. These competences must be acquired, partly, during the initial teacher education. This research aims to compare teacher competences perceptions between tutors and students in a degree of Physical Activity and Sports Science. A self-administered assessment questionnaire was passed in 201415 to 65 degree's students in year four and another scale to 29 tutors. There are significant differences between tutors and students in 24 of the 46 competences of the questionnaire. The students gave a higher average score than tutors in all competences except in 9 transversal competences. It is necessary to go deeply into the reasons produced behind this difference in the perception by conducting interviews and focus groups that provide more qualitative information.
\end{abstract}

Keywords: Teacher competences; initial teacher education; physical education 


\section{Resumo}

A competência docente refere-se à capacidade dos professores para enfrentar com sucesso os requisitos complexos que surgem no contexto educacional. Essas competências devem ser adquiridas, em parte, durante a formação inicial dos professores. Esta pesquisa tem como objetivo comparar como os professores e os alunos percebem o desenvolvimento de competências docentes em um curso de Ciências da Atividade Física e do Esporte. Durante o período 2014-15 foi aplicada uma escala de classificação autoadministrada a 65 alunos do $4^{\circ}$ ano e a 29 professores/as. Existem diferenças significativas entre professores e alunos em 24 das 46 competências do questionário. Em todas as competências os alunos apresentaram uma pontuação média mais elevada do que os professores, exceto em 9 competências transversais. É necessário aprofundar as razões que estão por trás dessa diferença de percepção realizando entrevistas e grupos focais para que possam fornecer informações mais qualitativas.

Palavras-chave: Competências docentes; formação inicial de professores; educação física

\section{Introducción}

El concepto de competencia es multidimensional y depende del contexto de los usuarios (Mulder, Weilgel \& Collins, 2007). Desde el ámbito educativo se entiende ésta como una intervención eficaz en diferentes ámbitos de la vida a través de acciones en las que se movilizan al mismo tiempo y de forma interrelacionada, conocimientos, procedimientos y actitudes (Zabala \& Arnau, 2008).

La competencia docente hace referencia a la capacidad del profesorado para resolver con éxito los problemas que se plantean en contextos educativos. Ésta debe ser adquirida durante la formación inicial del profesorado (en adelante FIP) y más tarde durante el propio ejercicio profesional. Por tanto, la formación inicial universitaria desempeñará un papel de primer orden en la configuración de la “identidad profesional de base”, dependiendo ésta de cómo se aprendan los conocimientos teóricos y los modelos de enseñanza y de cómo se adquiera una primera visión de la práctica profesional (Bolívar, 2008).

¿Perciben del mismo modo alumnado y profesorado el desarrollo de competencias docentes? El caso de un grado de ciencias de la actividad física y el deporte 
Por esta razón, resultará interesante conocer las diferencias de percepción entre profesorado y alumnado sobre las competencias docentes que se desarrollan en el grado. La peculiaridad del caso estudiado reside en ser un grado generalista, no especializado en la enseñanza de la Educación Física (EF), pues esta corresponde al Máster de Formación del Profesorado de Secundaria, Bachillerato y Ciclos Formativos.

\section{Método}

Se pasó una escala de valoración auto administrada a 65 estudiantes de $4^{\circ}$ curso que habían finalizado el período de prácticas (61\% de los estudiantes matriculados en el prácticum) y otra a 29 profesores y profesoras (91\% del total del profesorado que habían impartido docencia a dichos estudiantes a lo largo de los cuatro años de formación) de un grado de Ciencias de la Actividad Física y el Deporte (CAFyD).

Las escalas eran tipo Likert de 0-4 (0 nada, 1 poco, 2 medianamente, 3 mucho, 4 muchísimo). Mostraba un total de 46 competencias específicas y transversales relacionadas con la profesión docente validada en el proyecto $\mathrm{I}+\mathrm{D}+\mathrm{i}$ nacional (EDU 2013-42024-R) desarrollado por investigadores de la Red Nacional de Evaluación Formativa y Compartida.

Se calcularon las medias (M) y desviación estándar (DE). Se compararon las medias a partir de la prueba no paramétrica U de Mann-Witney para dos grupos independientes (profesores vs alumnos). Todos los análisis se realizaron con el programa SPSS 17,0. El nivel de significación estadística se estableció en $\mathrm{p}<0.05$.

\section{Resultados y discusión}

Los resultados obtenidos en la investigación muestran que:

1. El alumnado valora 30 competencias con más de 2 puntos. El profesorado valora 16 con más de 2 puntos (Tabla 1).

Tabla 1. Resultados de las valoraciones en alumnado y profesorado

\begin{tabular}{cccc}
\hline Alumnado & Valoración competencias & Profesorado & Valoración competencias \\
\hline Media & 2,31 & Media & 1,80 \\
DE & 0,54 & DE & 0,25 \\
\hline
\end{tabular}

¿Perciben del mismo modo alumnado y profesorado el desarrollo de competencias docentes? El caso de un grado de ciencias de la actividad física y el deporte 
2. Existen diferencias significativas entre profesorado y alumnado en 24 de las 46 competencias que contempla el cuestionario, destacando las siguientes $(p<0,001)$ (Tabla 2).

Tabla 2. Competencias con diferencias significativas $(p<0,001)$ entre alumnado y profesorado

\begin{tabular}{|c|c|c|c|c|}
\hline \multirow[t]{2}{*}{ Competencias } & \multicolumn{2}{|c|}{$\begin{array}{c}\text { Alumnado } \\
(n=65)\end{array}$} & \multicolumn{2}{|c|}{$\begin{array}{c}\text { Profesorado } \\
(n=29)\end{array}$} \\
\hline & $M$ & $D E$ & $M$ & $D E$ \\
\hline $\begin{array}{l}\text { Elaborar y poner en práctica programas de EF que faciliten la } \\
\text { inclusión efectiva de alumnos con NEE }\end{array}$ & 1,89 & 0,79 & 1,08 & 1,23 \\
\hline $\begin{array}{l}\text { Conocer y promover las diferentes manifestaciones motrices que } \\
\text { forman parte de la cultura tradicional }\end{array}$ & 2,17 & 0,98 & 1,12 & 1,11 \\
\hline $\begin{array}{l}\text { Conocer las capacidades físicas y los factores que determinan su } \\
\text { evaluación y saber aplicar sus fundamentos técnicos específicos }\end{array}$ & 2,75 & 0,77 & 1,77 & 1,42 \\
\hline $\begin{array}{l}\text { Conocer los fundamentos biológicos y fisiológicos del cuerpo } \\
\text { humano en relación con la A.F. }\end{array}$ & 2,82 & 0,83 & 1,50 & 1,42 \\
\hline $\begin{array}{l}\text { Conocer los fundamentos de la expresión corporal y } \\
\text { comunicación no verbal }\end{array}$ & 2,11 & 0,87 & 1,15 & 1,22 \\
\hline Disponer de estrategias de aplicación de los elementos de salud & 2,23 & 0,91 & 1,12 & 1,18 \\
\hline $\begin{array}{l}\text { Saber aplicar los fundamentos de las actividades físicas del medio } \\
\text { natural }\end{array}$ & 2,35 & 0,78 & 0,88 & 1,18 \\
\hline Dar respuesta a la diversidad en las prácticas de educación física & 2,35 & 0,93 & 1,42 & 1,33 \\
\hline $\begin{array}{l}\text { Identificar y prevenir riesgos que se derivan para la salud de la } \\
\text { práctica de actividades física inadecuadas }\end{array}$ & 2,77 & 0,93 & 1,92 & 1,13 \\
\hline Conocer características de la organización de los centros & 1,63 & 0,84 & 0,58 & 0,90 \\
\hline Elaborar propuestas de cambio del a realidad educativa & 1,65 & 0,89 & 1,00 & 1,36 \\
\hline $\begin{array}{l}\text { Elaborar y poner en práctica estrategias de atención a la } \\
\text { diversidad }\end{array}$ & 2,03 & 0,79 & 1,12 & 1,11 \\
\hline Implicar al alumnado en su aprendizaje y en la vida del centro & 2,14 & 0,79 & 1,31 & 1,12 \\
\hline Informar e implicar a las familias & 1,40 & 1,14 & 0,46 & 0,76 \\
\hline
\end{tabular}

3. En todas las competencias el alumnado proporcionó una puntuación media más elevada que el profesorado, excepto en 9, todas ellas transversales. El alumnado percibe su grado de competencia docente como más elevado en comparación a la percepción que tiene el profesorado sobre cuánto las desarrolla en las asignaturas que imparte. Esta diferencia puede ser debida a que la perspectiva del alumnado es global y la del profesorado parcial al haber profesores que imparten asignaturas con nulo o escaso componente didáctico. Estos resultados se contraponen a los obtenidos en el estudio de Gutiérrez-García et al (2011) realizado con alumnado de Magisterio, donde el profesorado es quién tiene una percepción mayor de su desarrollo. Sería necesario profundizar en los aspectos contextuales para determinar los motivos de estas diferencias.

¿Perciben del mismo modo alumnado y profesorado el desarrollo de competencias docentes? El caso de un grado de ciencias de la actividad física y el deporte 


\section{Conclusiones}

El alumnado tiene una percepción elevada sobre su competencia docente a pesar de tratarse de un grado generalista. Las posibles razones podrían ser: (1) el alumnado no es consciente de lo que todavía puede llegar a aprender; (2) muchos estudiantes de CAFyD tienen experiencias profesionales durante la carrera o provienen de ciclos formativos; (3) la carrera siempre se ha caracterizado por tener un componente didáctico elevado; (4) las características y la forma de enseñar las diferentes asignaturas pueden constituir un modelo competencial que arraiga en los estudiantes; (5) las metodologías vivenciadas a lo largo de su escolaridad y vida deportiva suponen modelos didácticos que han ido influyendo en la configuración de su identidad profesional de base.

Resulta necesario seguir profundizando en las razones que se hallan tras esta diferencia de percepción entre alumnado y profesorado. Para ello, sería necesario realizar entrevistas y grupos focales a todos los agentes participantes que aporten información más cualitativa.

\section{Referencias}

Bolívar, A. (2008). El discurso de las competencias en España: educación básica y educación superior. Red U. Revista de Docencia Universitaria, 2, 1-23.

Gutiérrez-García, C., Pérez-Pueyo, Á., Pérez-Gutiérrez, M., \& Palacios-Picos, A. (2011). Percepciones de profesores y alumnos sobre la enseñanza, evaluación y desarrollo de competencias en estudios universitarios de formación del profesorado. Cultura y Educación, 23(4), 499-514.

Mulder, M., Weigel, T., \& Collins, K. (2007). The concept of competence in the development of vocational education and training in selected EU member status: a critical analysis. Journal of Vocational Education \& Training, 59(1), 67-88.

Zabala, A., \& Arnau, L. (2008). 11 Ideas clave: como aprender y enseñar competencias. Barcelona España: Graó. 